\title{
PRÁTICAS CONTRACEPTIVAS E PREVENÇÃO DE DOENÇAS SEXUALMENTE TRANSMISSÍVEIS ENTRE ACADÊMICOS DE ENFERMAGEM
}

\author{
Lillia Michely Nunes Abreu¹, Anamária Serra Tavares²
}

RESUMO: Este estudo descritivo com abordagem quantitativa teve por objetivos investigar as condutas e práticas de contracepção e prevenção de doenças sexualmente transmissíveis entre acadêmicos de enfermagem, identificar os métodos contraceptivos utilizados e descrever comportamentos de risco. Os dados foram obtidos através de questionário aplicado a 135 estudantes entre maio e junho de 2011, e analisados pela estatística descritiva com frequência absoluta e porcentagem. A amostra foi constituída, em sua maioria, por jovens do sexo feminino, solteiros e com idade entre 20 a 23 anos. O preservativo masculino e o anticoncepcional oral foram os métodos mais relatados, usados separadamente ou combinados. Verificou-se precocidade das relações sexuais e práticas sexuais isentas do uso de preservativo, o que resultou em 5,9\% dos estudantes que contraíram doença sexualmente transmissível. Constatou-se que acadêmicos de enfermagem apresentam precariedade de condutas contraceptivas e de prevenção de doenças sexualmente transmissíveis. PALAVRAS-CHAVE: Anticoncepção; Comportamento sexual; Doenças sexualmente transmissíveis; Estudantes de enfermagem.

\section{CONTRACEPTIVE PRACTICES AND THE PREVENTION OF SEXUALLY- TRANSMITTED DISEASES AMONG NURSING ACADEMICS}

\begin{abstract}
This descriptive study with a quantitative approach aimed to investigate conduct and practices in contraception and the prevention of sexually-transmitted diseases among nursing academics, identify the methods of contraception used and to describe risky behaviors. The data was obtained via a questionnaire applied to 135 students between May and June 2011, and was analyzed by descriptive statistics with absolute frequency and percentages. The sample was mainly made up of single young women, aged between 20 and 23. The masculine condom and the oral contraceptive pill were the most-related methods, used separately or combined. Early initiation of sexual relations and practices without the use of condoms was noted, which resulted in 5,9\% of the students having contracted a sexually-transmitted disease. It was observed that nursing academics' conduct, where contraception and prevention of sexually-transmitted diseases are concerned, is precarious.

KEYWORDS: Contraception; Sexual behavior; Sexually-transmitted diseases; Student nurses.

\section{PRÁCTICAS CONTRACEPTIVAS Y PREVENCIÓN DE ENFERMEDADES SEXUALMENTE TRANSMISIBLES ENTRE ACADÉMICOS DE ENFERMERÍA}

RESUMEN: Este estudio descriptivo con abordaje cuantitativo tuvo por objetivos investigar las conductas y prácticas de contracepción y prevención de enfermedades sexualmente transmisibles entre académicos de enfermería, identificar los métodos contraceptivos utilizados y describir comportamientos de riesgo. Los datos fueron obtenidos por medio de cuestionario aplicado a 135 estudiantes entre mayo y junio de 2011, y analizados por la estadística descriptiva con frecuencia absoluta y porcentaje. La muestra se constituyó, en su mayoría, por jóvenes del sexo femenino, solteros y con edad entre 20 y 23 años. El preservativo masculino y el anticoncepcional oral fueron los métodos más relatados, usados separadamente o combinados. Se verificó precocidad de las relaciones sexuales y prácticas sexuales libres del uso de preservativo, lo que resultó en 5,9\% de los estudiantes que contrajeron enfermedad sexualmente transmisible. Se constató que académicos de enfermería presentan precariedad de conductas contraceptivas y de prevención de enfermedades sexualmente transmisibles. PALABRAS CLAVE: Anticoncepción; Comportamiento sexual; Enfermedades sexualmente transmisibles; Estudiantes de enfermería.

${ }^{1}$ Enfermeira. Especializanda em Enfermagem Obstétrica e Neonatal pelo Centro Universitário do Maranhão - UNICEUMA.
${ }^{2}$ Enfermeira. Especialista em Enfermagem Obstétrica e Neonatal. Supervisora técnica e Professora do UNICEUMA.

Autor correspondente:

Recebido: 10/01/2012

Lillia Michely Nunes Abreu Aprovado: 11/05/2012

Centro Universitário do Maranhão

Rua Josué Montello, 1 - 65075-120 -São Luís-MA-Brasil

E-mail: lilliaabreu@hotmail.com 


\section{INTRODUÇÃO}

A sexualidade é um componente fundamental da vida e engloba a reprodução, o amor e o prazer. É entendida como um conjunto de comportamentos e condutas que influenciam o ciclo de vida do ser humano, por estar diretamente relacionada à fatores biológicos e psicossociais ${ }^{(1)}$.

A liberdade sexual hoje existente deve-se, especialmente, à descoberta da pílula anticoncepcional, na década de 1960, o que proporcionou uma revolução em termos de saúde pública, controle da fecundidade e comportamento sexual. Posteriormente, em meados de 1980, o Planejamento Familiar tornou-se objeto de discussão entre vários segmentos da Sociedade e do Estado, possuindo, como objetivo primordial, proporcionar aos casais informação, assistência especializada e recursos necessários para que possam optar, livre e conscientemente, por ter, ou não, filhos, o número e o espaçamento entre eles, sem discriminação, coerção ou violência ${ }^{(2-3)}$.

A disponibilidade dos diversos métodos contraceptivos e o acesso à informação de qualidade são aspectos essenciais nos programas de planejamento familiar ${ }^{(4)}$. Entretanto, muitos jovens iniciam a vida sexual sem as orientações necessárias quanto aos métodos de prevenção, ficando expostos à gravidez indesejada, aborto, doenças sexualmente transmissíveis e à Síndrome da Imunodeficiência Adquirida (AIDS), que podem comprometer o projeto de vida, e até mesmo a própria vida ${ }^{(5)}$.

O excesso de confiança na invulnerabilidade, os tabus sociais e familiares e a obtenção de informação por intermédio de pessoas não qualificadas são fatores que podem resultar em práticas sexuais inseguras. Tais como, a não utilização de preservativo, a promiscuidade, a automedicação, as relações sexuais sem os cuidados e higiene necessários e a insegurança para solicitar ao parceiro o uso de métodos anticoncepcionais e de prevenção para as doenças sexualmente transmissíveis (DST) $)^{(6)}$.

Os métodos mais eficazes para a prevenção da gravidez não são, necessariamente, os mais efetivos para prevenir DST/AIDS. Dentre os difundidos, o preservativo é o único método eficaz que oferece dupla proteção, e que tem papel de destaque por proteger o usuário de doenças que podem ser transmitidas através das relações sexuais ${ }^{(2)}$.

Devido à sua magnitude e graves consequências, como infecções congênitas, câncer genital, e até mesmo a morte, as DST são consideradas um problema de saúde pública $^{(7)}$. Estudos mostram que o perfil epidemiológico da AIDS vem sofrendo transformações significativas, $o$ que nos leva a pensar em comportamentos de riscos e não em grupos de riscos, uma vez que essa doença vem propagando-se entre diversos segmentos, não ficando restrita a grupos específicos, como os homossexuais, o que corrobora a hipótese de heterossexualização, feminização e pauperização da epidemia ${ }^{(8-9)}$.

A educação em saúde é a principal estratégia na redução da incidência de gravidez indesejada e na transmissão de DST/AIDS, sendo também instrumento de informação e de conscientização para o autocuidado. Os profissionais de saúde, em especial de Enfermagem, devem considerar a particularidade de cada cliente, e oferecer informações de forma clara e objetiva sobre os diferentes métodos contraceptivos e de prevenção de $\operatorname{DST}^{(10)}$.

Considerando que na universidade, particularmente na área de saúde, os jovens têm maior acesso às informações acerca de contracepção e práticas sexuais inseguras, surgiu o interesse em pesquisar se os conhecimentos adquiridos na academia estariam sendo utilizados pelos estudantes para obter vida sexual sem riscos de ocorrer gravidez não planejada ou de adquirir DST/AIDS. Optou-se pelo curso de Enfermagem por oferecer acesso à informações de esfera biológica, relativas à anticoncepção e DST, as quais são acessíveis em disciplinas da grade curricular, entre essas Saúde da Mulher, Saúde Coletiva e Fisiologia.

Em razão do exposto, o estudo teve como objetivos investigar as condutas e práticas voltadas para a contracepção e prevenção de DST entre acadêmicos de Enfermagem, identificar os métodos contraceptivos por eles utilizados e descrever os comportamentos de risco para aquisição de DST e gravidez indesejada.

\section{MÉTODO}

Estudo descritivo e exploratório, de caráter quantitativo, realizado entre maio e junho de 2011, no Centro Universitário do Maranhão (UNICEUMA), Campus Renascença, localizado na capital São Luís.

A amostra foi constituída por 135 acadêmicos do Curso de Enfermagem. Calculou-se o tamanho amostral utilizando o sub-programa Statcalc do Epi Info 3.4.2, versão 2007, com os seguintes parâmetros: população de 895 acadêmicos de Enfermagem matriculados no primeiro semestre de 2011, nível de significância $(\alpha)$ de $5 \%$, erro tolerável de $8 \%$, e $10 \%$ de possíveis perdas. 
O cálculo baseou-se em uma prevalência de 81,4\% de uso de métodos contraceptivos entre universitárias, cuja iniciativa foi de ambos os parceiros ${ }^{(11)}$.

A amostragem foi estratificada por período em andamento, e aleatória dentro de cada período. Buscou-se manter a proporcionalidade de acadêmicos matriculados desde o primeiro ao oitavo período. Os critérios de inclusão no estudo foram: acadêmicos do Curso de Enfermagem, de ambos os sexos, regularmente matriculados no primeiro semestre de 2011 no UNICEUMA, que iniciaram atividade sexual e que aceitassem preencher o questionário proposto.

A coleta de dados foi realizada durante os intervalos de aula, utilizando-se um questionário composto por 22 questões fechadas, distribuídas em cinco seções: características sociodemográficas, características reprodutivas, práticas contraceptivas, comportamentos sexuais e ocorrência de DST. Os dados foram apresentados em forma de tabelas, analisados pela estatística descritiva com frequência absoluta e porcentagem, através do programa Epi Info, versão 3.4.2, e discutidos a partir da literatura pertinente.

A fim de garantir o cumprimento das questões éticas, o estudo foi previamente aprovado pelo Comitê de Ética em Pesquisa do UNICEUMA, sob protocolo n. 274/11. A pesquisa foi realizada mediante apresentação e assinatura do Termo de Consentimento Livre e Esclarecido com garantia do sigilo e anonimato das informações, seguindo os princípios éticos e legais que envolvem os seres humanos e a pesquisa, de acordo com a Resolução 196/96 do Conselho Nacional de Saúde.

\section{RESULTADOS}

\section{Caracterização dos Acadêmicos de Enfermagem e a Vida Reprodutiva}

A amostra foi constituída, predominantemente, por jovens do sexo feminino (74,8\%), com idade entre 20 a 23 anos $(29,6 \%)$ e renda familiar de quatro a seis salários mínimos (39,2\%). Quanto ao estado civil, 72,6\% dos alunos eram solteiros (77 mulheres e 21 homens) e os demais casados (20\%), unidos consensualmente (5,2\%) e divorciados (2,2\%).

Em relação às características reprodutivas, a tabela 1 demonstra que $24,4 \%$ dos acadêmicos (27 mulheres e seis homens) vivenciaram uma gravidez; a maioria das mulheres (14) relatou uma gravidez e quatro homens afirmaram ter engravidado suas parceiras uma vez.

O não uso de método anticoncepcional (MAC) foi a causa de $45,4 \%$ dos casos de gravidez; $21,2 \%$ desejavam engravidar, $18,2 \%$ não usavam preservativo em todas as relações sexuais, 9,1\% pararam de usar o MAC e 6,1\% relataram outros motivos, dentre eles a ruptura do preservativo masculino.

\section{Práticas Contraceptivas}

A maioria dos acadêmicos não utilizou contraceptivo na primeira relação sexual, representando 39,3\% do total, o que indica a alta vulnerabilidade em relação à gravidez indesejada e às DST/AIDS.

Quanto às razões para o não uso de contraceptivo na primeira prática sexual, $30,6 \%$ revelaram não ter se

Tabela 1 - Características reprodutivas dos acadêmicos de enfermagem, segundo o sexo. São Luís, 2011

\begin{tabular}{lcccccc}
\hline Características Reprodutivas & \multicolumn{2}{c}{ Sexo Feminino } & \multicolumn{2}{c}{ Sexo Masculino } & \multicolumn{2}{c}{ Total } \\
& $\mathbf{N}$ & $\mathbf{\%}$ & $\mathbf{N}$ & $\mathbf{\%}$ & $\mathbf{N}$ & $\mathbf{\%}$ \\
\hline Ocorrência de Gravidez & & & & & & \\
Sim & 27 & 26,7 & 6 & 17,6 & 33 & 24,4 \\
Não & 74 & 73,3 & 28 & 82,4 & 102 & 75,6 \\
Total & 101 & 100 & 34 & 100 & 135 & 100 \\
Número de Gravidez & & & & & & \\
1 & 14 & 51,9 & 4 & 66,7 & 18 & 54,6 \\
2 & 9 & 33,3 & 2 & 33,3 & 11 & 33,3 \\
3 & 4 & 14,8 & - & - & 4 & 12,1 \\
Total & 27 & 100 & 6 & 100 & 33 & 100 \\
Resultado da Gravidez & & & & & & \\
Nascido vivo & 19 & 70,4 & 4 & 66,7 & 23 & 69,7 \\
Aborto provocado & 5 & 18,5 & 2 & 33,3 & 7 & 21,2 \\
Aborto espontâneo & 1 & 3,7 & - & - & 1 & 3 \\
Gravidez em curso & 2 & 7,4 & - & - & 2 & 6,1 \\
Total & 27 & 100 & 6 & 100 & 33 & 100 \\
\hline
\end{tabular}


lembrado de usar contraceptivo; $24,5 \%$ não se preocuparam com gravidez; $20,4 \%$ relataram que o parceiro não quis usar preservativo; $14,3 \%$ não possuíam preservativo no momento do ato sexual; $6,1 \%$ não sabiam como e qual usar; e $4,1 \%$ tiveram outros motivos.

A tabela 3 apresenta os métodos contraceptivos que os acadêmicos estavam usando no momento da pesquisa; $65,1 \%$ utilizavam apenas um método e $28,2 \%$ usavam dois ou mais MAC; a dupla proteção foi observada por grande parte dos entrevistados. Os métodos mais cita-

Tabela 2 - Distribuição dos Acadêmicos de Enfermagem, segundo o método anticoncepcional utilizado na primeira relação sexual. São Luís, 2011

\begin{tabular}{lcc}
\hline $\begin{array}{l}\text { Método anticoncepcional utilizado } \\
\text { na primeira relação sexual }\end{array}$ & N & \% \\
\hline Coito interrompido & 13 & 9,6 \\
Coito interrompido + Pílula do dia & 6 & 4,4 \\
seguinte & 53 & 39,3 \\
Nenhum & 3 & 2,2 \\
Pílula & 9 & 6,7 \\
Pílula do dia seguinte & 49 & 36,3 \\
Preservativo masculino & 2 & 1,5 \\
Preservativo masculino + pílula & 135 & 100 \\
\hline
\end{tabular}

Tabela 3 - Distribuição dos Acadêmicos de Enfermagem, segundo o método contraceptivo utilizado. São Luís, 2011

\begin{tabular}{lcc}
\hline Método Contraceptivo Atual & N & \% \\
\hline Apenas Um Método & & \\
Preservativo masculino (Dupla pro- & 47 & 34,8 \\
teção*) & 21 & 15,5 \\
Pílula & 12 & 8,9 \\
Injeção & 2 & 1,5 \\
Coito interrompido & 1 & 0,7 \\
Tabelinha & 5 & 3,7 \\
Laqueadura & 88 & 65,1 \\
Subtotal & & \\
Dois ou Mais Métodos & 29 & 21,5 \\
Dupla proteção* & 7 & 5,2 \\
Métodos de alta eficácia** combinados & 2 & 1,5 \\
com métodos de baixa eficácia & 28,2 \\
Métodos de baixa eficácia*** & 9 & 6,7 \\
Subtotal & 135 & 100,0 \\
Nenhum & Total
\end{tabular}

*Uso do preservativo ou da sua combinação com outro método anticoncepcional, promovendo, simultaneamente, a proteção contra gravidez e DST/AIDS.

**Pílula e injeção.

***Coito interrompido e tabelinha. dos foram o preservativo masculino de forma isolada $(34,8 \%)$ ou combinada com outros métodos $(21,5 \%)$. Métodos comportamentais, como o coito interrompido $(1,5 \%)$ e a tabelinha $(0,7 \%)$, foram pouco mencionados.

Entre os entrevistados, $50,8 \%$ utilizavam o contraceptivo por indicação de um profissional de saúde; $16,7 \%$ revelaram que não receberam indicação sobre o método usado; $13,5 \%$ receberam influência da família; $11,1 \%$ do parceiro; e 7,9\% usavam o contraceptivo por indicação de amigo.

Ao serem questionados sobre o motivo que levou à escolha do atual contraceptivo, $56,4 \%$ dos acadêmicos referiram pela prevenção simultânea de gravidez e DST/AIDS, 22,2\% referiram eficácia do método; $14,3 \%$ relataram preocupação em prevenir apenas uma gravidez indesejada e 7,1\% referiram a prevenção de DST/AIDS.

\section{O Comportamento Sexual dos Acadêmicos de Enfermagem}

A prática sexual deu-se mais precocemente entre os homens. A maioria teve sua primeira relação sexual entre 12 e 15 anos (47,1\%), enquanto que as mulheres entre 16 e 19 anos (51,5\%). Ambos os sexos afirmaram manter, no momento da pesquisa, relações sexuais com parceiros fixos, representando $63,7 \%$ dos indivíduos. Em relação ao número de parceiros, observou-se que a monoparceria foi pouco relatada (20\%); a maioria dos homens revelou 10 ou mais parceiras $(35,3 \%)$ desde a iniciação sexual, e as mulheres entre dois a cinco parceiros (55,5\%) (Tabela 4).

Outro comportamento de risco observado foi a baixa frequência do uso de preservativo nas relações sexuais; os participantes responderam, em sua maioria, que "às vezes" usam o preservativo $(69,6 \%)$, seguidos daqueles que utilizam em todas as relações sexuais $(23 \%)$ e dos que nunca usam $(7,4 \%)$. As justificativas mais citadas pelas mulheres para tal comportamento foram a união estável $(32,5 \%)$ e a confiança no parceiro $(20 \%)$; entre os homens foram a interferência no prazer $(37,5 \%)$ ou não gostar de usá-lo (33,3\%).

Em relação à ocorrência de DST/AIDS, identificou-se que $90,4 \%$ nunca contraíram doenças; $5,9 \%$ já foram contaminados durante o ato sexual e 3,7\% não sabem se já foram infectados. Entre os que contraíram DST/AIDS, três $(37,5 \%)$ relataram o herpes genital, dois $(25 \%)$ gonorreia e um $(12,5 \%)$ Papilomavírus Humano, e dois $(25,0 \%)$ relataram outras DST (tricomoníase e clamídia). 
Tabela 4 - Caracterização do comportamento sexual dos Acadêmicos de Enfermagem, segundo o sexo. São Luís, 2011

\begin{tabular}{lcccccc}
\hline \multicolumn{1}{c}{ Comportamento Sexual } & \multicolumn{2}{c}{ Sexo Feminino } & \multicolumn{2}{c}{ Sexo Masculino } & \multicolumn{2}{c}{ Total } \\
& $\mathbf{N}$ & $\mathbf{\%}$ & $\mathbf{N}$ & $\mathbf{\%}$ & $\mathbf{N}$ & $\mathbf{\%}$ \\
\hline Idade da iniciação sexual & 1 & 1 & 3 & 8,8 & 4 & 3 \\
$<12$ anos & 19 & 18,8 & 16 & 47,1 & 35 & 25,9 \\
12 a 15 anos & 52 & 51,5 & 15 & 44,1 & 67 & 49,6 \\
16 a 19 anos & 24 & 23,8 & - & - & 24 & 17,8 \\
20 a 23 anos & 5 & 4,9 & - & - & 5 & 3,7 \\
24 a 27 anos & 101 & 100 & 34 & 100 & 135 & 100 \\
Total & & & & & & \\
Parceiro fixo & 68 & 67,3 & 18 & 52,9 & 86 & 63,7 \\
Sim & 33 & 32,7 & 16 & 47,1 & 49 & 36,3 \\
Não & 101 & 100 & 34 & 100 & 135 & 100 \\
Total & 25 & 24,7 & 2 & & & \\
Número de parceiros desde a iniciação sexual & & & 5,9 & 27 & 20 \\
1 & 56 & 55,5 & 9 & 26,4 & 65 & 48,1 \\
2 a 5 & 16 & 15,8 & 11 & 32,4 & 27 & 20 \\
6 a 9 & 4 & 4 & 12 & 35,3 & 16 & 11,9 \\
10 ou mais & 101 & 100 & 34 & 100 & 135 & 100 \\
Total & & & & & & \\
Uso do preservativo & 9 & 8,9 & 1 & 2,9 & 10 & 7,4 \\
Nunca & 71 & 70,3 & 23 & 67,7 & 94 & 69,6 \\
Às vezes & 21 & 20,8 & 10 & 29,4 & 31 & 23 \\
Sempre & 101 & 100 & 34 & 100 & 135 & 100 \\
Total & & & &
\end{tabular}

\section{DISCUSSÃO}

O perfil sociodemográfico apresentado nesta pesquisa corrobora os achados de outro estudo que tratam da saúde sexual de universitários, em que se destacam o predomínio de jovens solteiros, do sexo feminino, na faixa etária de 20 a $22 \operatorname{anos}^{(1)}$.

Trinta e três gestações foram mencionadas pelo grupo pesquisado, das quais 7 foram interrompidas por aborto provocado, revelando a falta de planejamento familiar e de gravidez indesejada. A maioria das gestações ocorreu devido ao não uso de métodos contraceptivos. Esse fato foi evidenciado em outro estudo no qual $57,6 \%$ das adolescentes engravidaram porque não se preveniram, outros fatores que levaram à gravidez foram o desejo de engravidar e a resistência dos familiares em oferecer informações aos adolescentes sobre práticas sexuais ${ }^{(12)}$.

Quanto ao número de gestações, a maioria dos acadêmicos de enfermagem vivenciou apenas uma gestação. A redução significativa nas taxas de fecundidade reflete as mudanças que vêm ocorrendo no Brasil, em especial com o aumento da escolaridade feminina, inserção da mulher no mercado de trabalho e do avanço tecnológico em relação às opções contraceptivas $^{(13)}$. Em 1970, as mulheres tinham em média 5,8 filhos, passando para 2,3 filhos em 2000, seguindo uma tendência de diminuição nos anos posteriores ${ }^{(14)}$.

O estudo mostrou um aumento no uso de contraceptivos quando comparado com a primeira prática sexual, com predominância do preservativo masculino e do anticoncepcional oral. De forma semelhante, pesquisa realizada com 952 estudantes de graduação de uma universidade pública estadual localizada na Cidade de São Paulo, revelou a predominância do preservativo e da pílula, usados separadamente ou em combinação ${ }^{(15)}$. O preservativo contribui para reduzir o risco de infecções adquiridas através da exposição à região vaginal, vulvar e anal. A sua eficácia está diretamente associada ao uso correto e regular, bem como à qualidade do preservativo ${ }^{(5)}$.

Verificou-se no estudo a baixa expressividade dos métodos comportamentais, como o coito interrompido. Isto evidencia que os acadêmicos conhecem sua baixa eficácia, a qual está relacionada com a possível eliminação de espermatozoides no líquido pré-ejaculatório e a necessidade de autocontrole por parte do homem no momento da ejaculação ${ }^{(1)}$. 
A pesquisa revelou a multiparceria sexual e a precocidade das relações sexuais, sendo que os homens iniciaram a vida sexual mais cedo do que as mulheres. Pesquisa sobre conhecimentos, atitudes e práticas na população brasileira de 15 a 64 anos, realizada em 2008, mostrou a diferença de comportamento entre homens e mulheres; $36,9 \%$ dos homens tiveram sua primeira relação antes dos 15 anos, enquanto que este índice cai para $17 \%$ entre as mulheres ${ }^{(16)}$. Quanto mais cedo ocorrer a primeira relação sexual, maiores serão as chances de engravidar, devido o desconhecimento de práticas preventivas, pelo baixo poder de compra de contraceptivos e receio na busca pelos serviços de saúde ${ }^{(17)}$.

No que concerne à regularidade do uso do preservativo nas relações sexuais, observou-se que a maioria dos estudantes, apesar de citar o preservativo como método de escolha, não o utiliza em todas as práticas sexuais, revelando carências nos aspectos preventivos. Acredita-se que relações esporádicas, confiança no parceiro e crenças na redução da sensibilidade durante $\mathrm{o}$ ato sexual sejam fatores que promovem o não uso de preservativo ${ }^{(5)}$.

O estudo revelou um número reduzido de acadêmicos $(5,9 \%)$ que afirmaram histórico de DST/AIDS, com maior relato de herpes genital. Vale ressaltar que a multiplicidade de parceiros, o sexo oral e anal por expor a uma carga virótica e bacteriana potencialmente maior, e o não uso do preservativo, são fatores de riscos que suscetibilizam os indivíduos a essas doenças ${ }^{(18)}$.

Os resultados mostram que, apesar dos acadêmicos apresentarem condutas contraceptivas e mencionarem o preservativo como método de escolha, não os adotam em todas as relações sexuais, revelando alta vulnerabilidade à gestação não planejada e às DST/AIDS.

\section{CONCLUSÕES}

O estudo revelou um aumento do uso atual de contraceptivos quando comparado com a primeira relação sexual; o preservativo masculino e o anticoncepcional oral foram os métodos mais relatados pelo grupo estudado, usados separadamente ou combinados. Os comportamentos sexuais de risco observados foram a precocidade das relações sexuais, a multiplicidade de parceiros e práticas sexuais isentas do uso de preservativo.

Os acadêmicos de enfermagem, apesar de apresentarem práticas voltadas para a prevenção de gravidez indesejada e DST/AIDS, estas são vivenciadas precariamente em alguns aspectos preventivos, principal- mente no que concerne à frequência do uso de preservativo nas relações sexuais. Assim, a escolaridade e o conhecimento sobre práticas sexuais inseguras não implica, necessariamente, no uso regular dos métodos contraceptivos.

Torna-se necessário estabelecer medidas de ensino e discussões no âmbito da sexualidade, com vistas à orientação dos acadêmicos quanto aos cuidados com a saúde sexual, a fim de reduzir o número de gravidez indesejada. Também, para prevenir as DST nessa população, enfatizando ainda o papel de educadores e multiplicadores de saúde que irão desempenhar como futuros enfermeiros.

\section{REFERÊNCIAS}

1. Falcão Júnior JSP, Rabelo STO, Lopes EM, Freitas LV, Pinheiro AKB, Ximenes LB. Perfil e práticas sexuais de universitários da área da saúde. Esc. Anna Nery. 2007;11(1):58-65.

2. Leite MTF, Costa AVS, Carvalho KAC, Melo RLR, Nunes BMTV, Nogueira LT. Saber e prática contraceptiva e prevenção de DST/HIV/AIDS em universitários da área da saúde. Rev Bras Enferm. 2007;60(4):434-8.

3. Inagaki ADM, Santos MD, Abud ACF, Gonçalves LLC, Daltro AST. Práticas contraceptivas entre acadêmicos de enfermagem de uma universidade federal. Rev. enferm. UERJ. 2007;15(4):563-8.

4. Martins LBM, Costa-Paiva L, Osis MJD, Sousa MH, Pinto Neto AM, Tadini V. Conhecimento sobre métodos anticoncepcionais por estudantes adolescentes. Rev. Saúde Pública. 2006;40(1):57-64.

5. Alves AS, Lopes MHBM. Uso de métodos anticoncepcionais entre adolescentes universitários. Rev Bras Enferm. 2008;61(2):170-7.

6. Rabelo STO, Falcão Júnior JSP, Freitas LV, Lopes EM, Pinheiro AKB, Aquino PS, et al. Gravidez e DST: práticas preventivas entre universitários. DST, j. bras. doenças sex. transm. 2006;18(2):148-55.

7. Ministério da Saúde (BR). Secretaria de Vigilância em Saúde Programa Nacional de DST e Aids. Manual de controle das doenças sexualmente transmissíveis. $4^{\mathrm{a}}$ ed. Brasília: Ministério da Saúde; 2006.

8. Brito AM, Castilho EA, Szwarcwald CL. AIDS e infecção pelo HIV no Brasil: uma epidemia multifacetada. Rev. 
Soc. Bras. Med. Trop. 2001;34(2):207-17.

9. Santos NJS, Tayra A, Silva SR, Buchalla CM, Laurenti R. A aids no Estado de São Paulo. As mudanças no perfil da epidemia e perspectivas da vigilância epidemiológica. Rev. bras. epidemiol. 2002;5(3):286-310.

10. Penaforte MCLF, Silva LR, Esteves APVS, Silva RF, Santos IMM, Silva MDB. Conhecimento, uso e escolha dos métodos contraceptivos por um grupo de mulheres de uma unidade básica de saúde em Teresópolis, RJ. Cogitare enferm. 2010;15(1):124-30.

11. Bastos MR, Borges ALV, Hoga LAK, Fernandes MP, Contin MV. Práticas contraceptivas entre jovens universitárias: o uso da anticoncepção de emergência. Texto contexto-enferm. 2008;17(3):447-56.

12. Ponte Júnior GM, Ximenes Neto FRG. Gravidez na adolescência no município de Santana do Acaraú Ceará - Brasil: uma análise das causas e riscos. Rev. eletr. enf. [Internet] 2004;6(1). [acesso em 08 jul 2011]. Disponível: http://www.revistas.ufg.br/index.php/fen/ article/view/802/911

13. Scavone L. Maternidade: transformações na família e nas relações de gênero. Interface-Comunic. Saude Educ. 2001;5(8):47-60.

14. Carvalho JAM, Garcia RA. O envelhecimento da população brasileira: um enfoque demográfico. Cad. Saúde Pública. 2003;19(3):725-33.

15. Pirotta KCM, Schor N. Intenções reprodutivas e práticas de regulação da fecundidade entre universitários. Rev. Saúde Pública. 2004;38(4):495-502.

16. Ministério da Saúde (BR). Secretaria de Vigilância em Saúde. Departamento de DST, Aids e hepatites virais. Pesquisa de conhecimentos, atitudes e práticas na população brasileira de 15 a 64 anos, 2008. Brasília: Ministério da Saúde; 2011.

17. Ximenes Neto FRG, Dias MSA, Rocha J, Cunha ICKO. Gravidez na adolescência: motivos e percepções de adolescentes. Rev Bras Enferm. 2007;60(3):279-85.

18. Smeltzer SC, Bare BG. Brunner e Suddarth: tratado de enfermagem médico-cirúrgico. $10^{\mathrm{a}}$ ed. Rio de Janeiro: Guanabara Koogan; 2004. 